\title{
Molecular confirmation of natural hybridisation between Melastoma sanguineum and M. malabathricum (Melastomataceae)
}

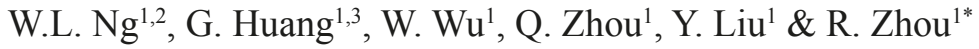 \\ ${ }^{1}$ State Key Laboratory of Biocontrol and Guangdong Provincial Key \\ Laboratory of Plant Resources, School of Life Sciences, \\ Sun Yat-sen University, 135 Xingang Xi Road, Haizhu District, \\ 510275 Guangzhou, Guangdong, China \\ *zhrench@mail.sysu.edu.cn \\ ${ }^{2}$ China-ASEAN College of Marine Sciences, Xiamen University Malaysia, \\ 43900 Sepang, Selangor, Malaysia \\ ${ }^{3}$ Guangzhou Landscaping Company, 510440 Guangzhou, \\ Guangdong, China
}

\begin{abstract}
The genus Melastoma (Melastomataceae) is known to have undergone rapid species radiation, and natural hybridisation has been observed to happen whenever two or more species co-occur. Many cases of natural hybridisation have been confirmed between Melastoma species in China, but only a few cases have been confirmed in Southeast Asia, which is where the majority of the diversity of the genus occurs, although hybrids have been suspected based on morphological intermediacy. Recently in Peninsular Malaysia, we observed co-occurring populations of Melastoma sanguineum Sims and M. malabathricum L., two of the most widely distributed species of Melastoma L. Many individuals with intermediate morphologies were also at the site. In this study, we used DNA sequence data of three partial nuclear genes and one chloroplast locus to determine the identity of the intermediate individuals. We found that the chloroplast haplotypes could be grouped by similarity to clusters corresponding to the two species, and the same individuals shared nuclear alleles from both clusters. Our findings revealed that, (1) the morphologically intermediate individuals are indeed hybrids of Melastoma sanguineum and M. malabathricum; (2) both F1 hybrids and further hybrid generations are present; (3) both species can act as pollen donor.
\end{abstract}

Keywords. Gene flow, hybrid, molecular evidence, species boundary, species radiation

\section{Introduction}

Melastoma L. (Melastomataceae) comprises species distributed mainly in Southeast Asia, with some extending to India, southern China, Japan, and northern Australia (Meyer, 2001). The number of species within the genus remains debatable, with claims ranging from 22 (Meyer, 2001) and 80-90 (Wong, 2016) to about 100 species (Chen, 1983). Using molecular clock analyses, Renner \& Meyer (2001) estimated that Melastoma diversified around 1 million years ago. However, due to their recent divergence and shared life history characteristics, such as partially overlapping flowering periods (Chen, 1984) and shared pollinators (Gross, 1993; Luo et al., 2008), 
hybridisation seems to happen readily between/among different species of Melastoma (natural hybridisation) whenever they co-occur (Dai et al., 2012; Liu et al., 2014; Zhou et al., 2017; Zou et al., 2017).

In China, about nine species of Melastoma have been documented (Chen, 1983). They all have the same chromosome number of $2 \mathrm{n}=24$ (Zhang et al., 2010). So far, natural hybridisation has been observed (through morphological intermediacy) for most of the species (R. Zhou, pers. obs.), and the status of some have been confirmed via molecular means (e.g. Dai et al., 2012; Liu et al., 2014; Zhou et al., 2017; Zou et al., 2017). While Southeast Asia is known to be the centre of Melastoma species diversity (Meyer, 2001), few reports of putative hybrids are available; in Borneo for example, a recent compilation by Wong (2016) documented 41 species, among which 40 species were endemic to the island, and only seven species were observed to form putative hybrids. All known species of Melastoma in China only flower at a given period of the year during the warmer months (Chen, 1984), which presents an important prezygotic barrier for natural hybridisation to happen. Southeast Asian Melastoma species, on the other hand, are known to flower throughout the year (R. Zhou \& W.L. Ng, pers. obs.), prompting us to believe that natural hybridisation should happen more easily (and hence more frequently) between co-occurring species. Only recently, two cases of natural hybridisation between Southeast Asian Melastoma have been confirmed through molecular studies - between M. malabathricum L. and $M$. laevifolium Merr., and between $M$. malabathricum and $M$. beccarianum Cogn. - both in Borneo (Cai et al., 2019; Wu et al., 2019). Confirming the true identities of such morphologically ambiguous taxa is important to further assist in the taxonomy of, as well as to understand the evolution within, Melastoma.

Melastoma malabathricum and $M$. sanguineum Sims are two of the most widely distributed species of Melastoma - the former extends from India to the western Pacific islands, and the latter from southern China to most parts of Southeast Asia (Meyer, 2001). On a recent survey in Kuala Selangor, Selangor, Malaysia, we discovered the two species growing in sympatry, with some individuals being morphologically intermediate between them, most notably in the indumentum (hair-like structures) of the hypanthium (floral cup), as shown in Figure 1. These individuals had hypanthium indumentum that is intermediate between the locally growing Melastoma malabathricum and M. sanguineum in terms of length, width, and curvature. Wong (2015) proposed the hypanthium indumentum as a reliable key characteristic in distinguishing the species of Melastoma. Morphologically intermediate individuals growing among sympatric populations of two or more species is often the first indication of hybridisation. In this study, we aimed to use molecular data to determine if the morphologically intermediate individuals were hybrids between Melastoma sanguineum and M. malabathricum. We sequenced one chloroplast DNA region as well as three partial nuclear genes to answer the following three questions: (1) Are the morphologically intermediate individuals hybrids between Melastoma sanguineum and M. malabathricum? (2) Are they F1 hybrids, or can individuals of further hybrid generations can be found? (3) Does hybridisation happen in one or both directions between the two species? 

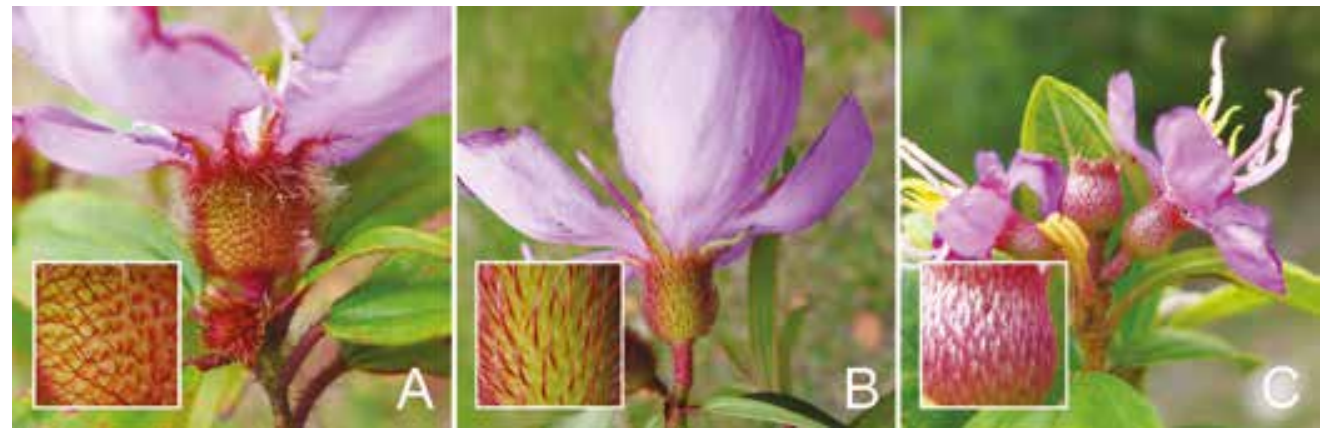

Fig. 1. Comparison of the hypanthia of Melastoma sanguineum, M. malabathricum and the putative hybrid. A. Melastoma sanguineum. B. Putative hybrid. C. M. malabathricum. The hypanthium indumentum in the putative hybrid is obviously intermediate between the putative parents in terms of the length, width, and curvature (inset). (Photos: Y. Liu).

\section{Materials and methods}

\section{Sampling and DNA extraction}

In Kuala Selangor, Selangor, Malaysia, we sampled 19 individuals of each putative parent, i.e. Melastoma sanguineum (KMS) and M. malabathricum (KMM), and 20 morphologically intermediate individuals, i.e. putative hybrids $(\mathrm{KMH})$, based on morphology. To provide further support for the morphologically identified putative parents, allopatric populations of each parental species were included as reference: Melastoma sanguineum from Kirilom Natural Park, Cambodia (JMS) and $M$. malabathricum from Genting Highlands, Malaysia (GMM). Eleven individuals were sampled from each of the reference populations. A summary of the sampling for this study is listed in Table 1. Voucher specimens of all samples used in this study (ZHOU2017001-ZHOU2017080) have been deposited at the Herbarium of Sun Yat-sen University (SYS). For molecular analysis, leaf material was sampled and dried in silica gel before DNA extraction. Total genomic DNA was extracted from approximately $50 \mathrm{mg}$ dried leaf material using the CTAB method and stored at $-20^{\circ} \mathrm{C}$ before further analyses.

\section{PCR and DNA sequencing}

Three partial low-copy nuclear genes: calmodulin (cam), triosephosphate isomerase (tpi), and peptide chain release factor 1 (pcrfl), as well as one chloroplast DNA locus ( $p s b \mathrm{~K}-p s b \mathrm{~L}$, which includes two partial genes and their intergenic region) were PCRamplified and sequenced from both directions for this study. Melastoma-specific primers of cam and tpi were according to our previous studies (Dai et al., 2012; Chao et al., 2014), while primers for pcrfl were initially designed for South American Melastomataceae (Reginato \& Michelangeli, 2016). The universal primers for the chloroplast locus $p s b \mathrm{~K}-p s b \mathrm{~L}$ were obtained from Meng et al. (1991).

PCR amplifications were performed in $20 \mu \mathrm{L}$ reaction mixtures, each containing 30-50 ng of genomic DNA, $1 \times$ PCR buffer, dNTP mixture $(0.2 \mathrm{mM}$ of each dNTP), 
Table 1. Sampling information for Melastoma spp. used in this study.

\begin{tabular}{llc}
\hline \multicolumn{1}{c}{ Taxon } & \multicolumn{1}{c}{ Locality (Population code) } & Sample size \\
\hline M. sanguineum & Kuala Selangor, Malaysia (KMS) & 19 \\
M. malabathricum & Kuala Selangor, Malaysia (KMM) & 19 \\
Putative hybrid & Kuala Selangor, Malaysia (KMH) & 20 \\
M. sanguineum & Kirilon Natural Park, Cambodia (JMS) & 11 \\
M. malabathricum & Genting Highlands, Malaysia (GMM) & 11 \\
\hline
\end{tabular}

$0.25 \mu \mathrm{M}$ of each primer, and 1.0 U of KOD-FX DNA polymerase (TOYOBO). The $\mathrm{PCR}$ reaction profile comprised an initial denaturation of $3 \mathrm{~min}$ at $95^{\circ} \mathrm{C}$; followed by 32 cycles of $30 \mathrm{~s}$ at $95^{\circ} \mathrm{C}, 30 \mathrm{~s}$ at $54^{\circ} \mathrm{C}$, and $2 \mathrm{~min}$ at $72^{\circ} \mathrm{C}$; and finally an extension step at $72^{\circ} \mathrm{C}$ for $7 \mathrm{~min}$. Purified PCR products were sequenced on an ABI 3730 DNA Analyzer (Applied Biosystems). DNA sequences that contained more than one polymorphic site were subjected to cloning using the pMD 18-T Vector cloning kit (Takara) to separate the haplotypes, and sequenced.

\section{Data analyses}

DNA sequences were assembled and aligned using SeqMan (DNASTAR Inc). DnaSP ver. 5 (Librado \& Rozas, 2009) was then used to phase the haplotypes. The sequences generated in this study were deposited in the NCBI GenBank database with the accession numbers MT118836-MT118967. The haplotype network for each locus was constructed using Network ver. 4.6 (www.fluxus-engineering.com), using the median joining algorithm (Bandelt et al., 1999). The algorithm combines the features of a maximum-parsimony heuristic algorithm and a minimum spanning tree search algorithm to resolve the relationships of the haplotypes.

Genetic structure analysis was performed through the Bayesian STRUCTURE ver. 2.3.3 (Pritchard et al., 2000), using the variable sites of the three nuclear loci to assign the sampled individuals to genetic clusters. To determine the optimal number of clusters, ten independent runs for each value of $K=1-10$ were conducted with $10^{5}$ burn-ins and $10^{6}$ Markov Chain Monte Carlo (MCMC) iterations using the admixture and correlated allele frequency models. The optimal $K$ value, was obtained with the $\Delta K$ statistics (Evanno et al., 2005) through Structure Harvester ver. 0.6.94 (Earl \& vonHoldt, 2012). Membership coefficients at each of the suggested number of clusters for each individual were then estimated across the ten independent runs using CLUMPP ver. 1.1.2 (Jakobsson \& Rosenberg, 2007). Finally, Distruct ver. 1.1 (Rosenberg, 2004) was used to generate a graphical display of the population structuring.

Theoretically, in diploid organisms, an offspring inherits one allele of a nDNA locus from each parent. A hybrid can therefore be detected from nDNA sequence data by recognising that it harbours haplotypes from two species. Furthermore, while an F1 
hybrid harbours haplotypes from both species at all tested loci, advanced hybridisation would produce individuals that show segregation of parental haplotypes across different loci. Chloroplast DNA, on the other hand, is maternally inherited in most plants (Mogensen, 1996), and so is useful for testing to see if hybridisation happens in one or both directions.

\section{Results}

\section{Haplotype analysis of chloroplast DNA locus}

The total aligned length of the chloroplast locus was 420 bp. As shown in Figure 2, there were four haplotypes among all the samples - two haplotypes were specific to the reference populations JMS and GMM, while the other two haplotypes were shared, each mainly by individuals of either KMS (H_1) or KMM (H_2). The putative hybrids had either of the two shared haplotypes. Interestingly, while the haplotypes were distinct for the two species in Kuala Selangor, the separation of haplotypes in the two species was not complete across all samples, which can be attributed to past hybridisation events, or incomplete lineage sorting.

\section{Haplotype analysis of nuclear DNA loci}

tpi: The total aligned length of tpi was $735 \mathrm{bp}$. A total of 25 haplotypes were observed for this locus, and two main clusters separated by eight mutational steps can be observed - one occupied mainly by haplotypes of Melastoma sanguineum and the other mainly by haplotypes of M. malabathricum. As shown in Figure 3A, samples of KMS had two haplotypes, JMS had eight haplotypes, KMM had eight haplotypes, GMM had ten haplotypes, while the putative hybrids had three haplotypes. Most of the putative hybrids share a haplotype with Melastoma sanguineum, while two haplotypes are unique (one closer to the $M$. sanguineum haplotype cluster and the other closer to the M. malabathricum haplotype cluster).

cam: The total aligned length of cam was $708 \mathrm{bp}$. A total of 43 haplotypes were observed for this locus, forming two main clusters occupied mainly by haplotypes of Melastoma sanguineum and of $M$. malabathricum. As shown in Figure 3B, samples of KMS had ten haplotypes, JMS had 15 haplotypes, KMM had seven haplotypes, GMM had nine haplotypes, while the putative hybrids had 15 haplotypes. The haplotypes of the putative hybrids are distributed between the Melastoma sanguineum and $M$. malabathricum clusters.

pcrf1: The total aligned length of pcrfl was $608 \mathrm{bp}$. A total of 33 haplotypes were obtained for this locus, and except for one shared haplotype, different haplotypes were observed for samples of Melastoma sanguineum and M. malabathricum. As shown in Figure 3C, samples of KMS had four haplotypes, JMS had nine haplotypes, KMM had 14 haplotypes, GMM had 13 haplotypes, while the putative hybrids had eight haplotypes. Except for one haplotype (H_11), the haplotypes of the putative hybrids are distributed between the Melastoma sanguineum and M. malabathricum clusters. 


\section{$p s b K-p s b L$}

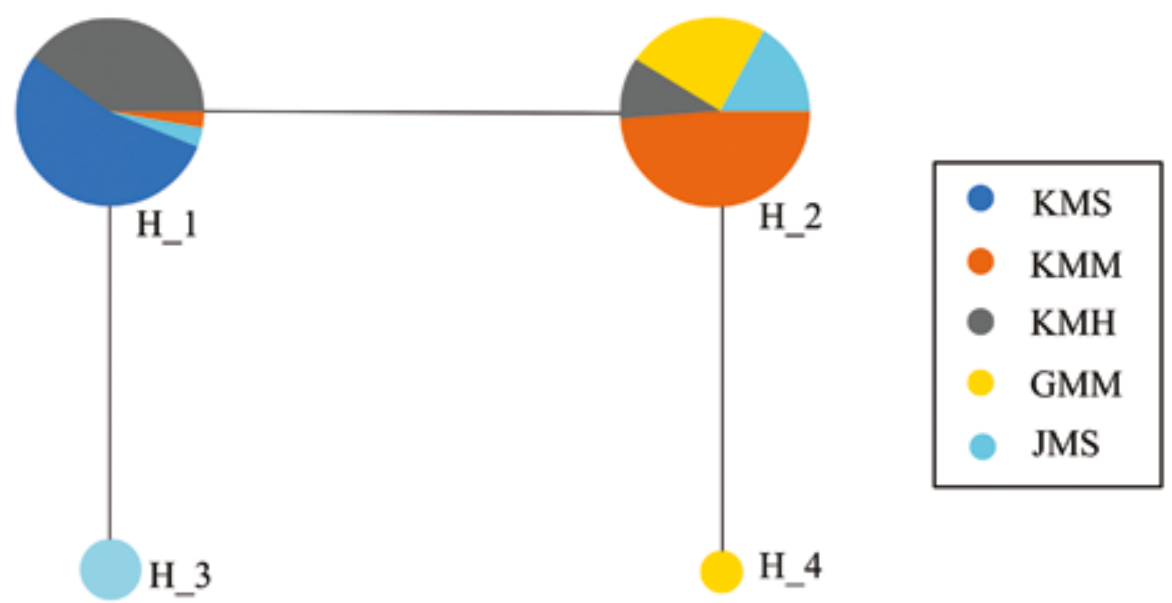

Fig. 2. Haplotype networks of chloroplast locus $p s b K-p s b L$. Haplotypes of each population/ taxon are shown in different colours. The sizes of the circles correspond to frequencies of the haplotypes. Haplotypes connected by lines are one mutational step from each other.

\section{Population structure analysis}

The Bayesian clustering-based STRUCTURE analysis yielded the highest $\Delta K$ value at $K=2$ (Figure $4 \mathrm{a}$ ), indicating that the individuals sampled in this study most likely belonged to two genetic clusters. It is noteworthy that while the $\Delta K$ analysis cannot distinguish between $K=1$ and $K=2$ (and so $K=1$ cannot be ruled out), high genetic differentiation $\left(\mathrm{F}_{\mathrm{ST}}>0.7\right.$; data not shown) was estimated between populations KMM and KMS, thus supporting $K=2$. Aside from two individuals from population KMS, the bar plot obtained for $K=2$ (Figure 4b) showed high probability of assignment of Melastoma sanguineum and M. malabathricum into separate clusters, across both the sympatric and allopatric populations. Most individuals of the putative hybrids $(\mathrm{KMH})$ displayed a mixture of genetic components from Melastoma sanguineum and M. malabathricum in varying degrees. The Bayesian clustering results thus supported the morphologically intermediate individuals as hybrids of the two species.

\section{Discussion}

From the haplotype networks, most of the haplotypes of Melastoma sanguineum and M. malabathricum clustered separately across populations at all the loci sequenced in this study. This and the Bayesian clustering analysis indicate that the two species are genetically distinct as suggested by morphological classification. At the nuclear DNA loci, the morphologically intermediate individuals harboured haplotypes that were identical or clustered with haplotypes from both Melastoma sanguineum 


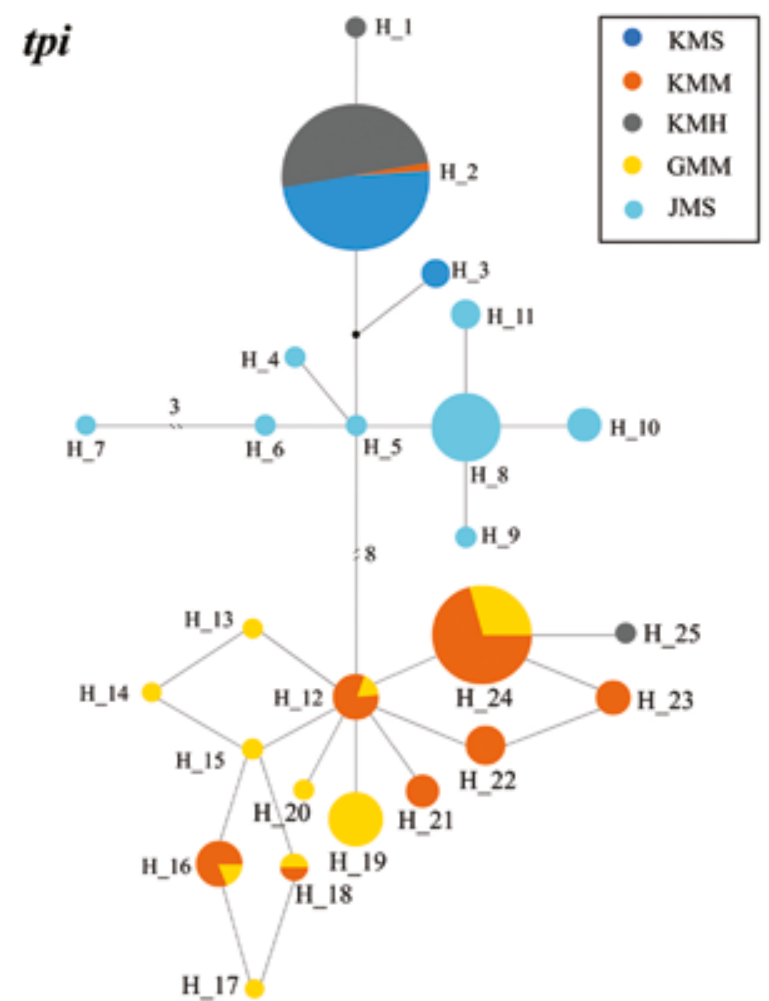

A

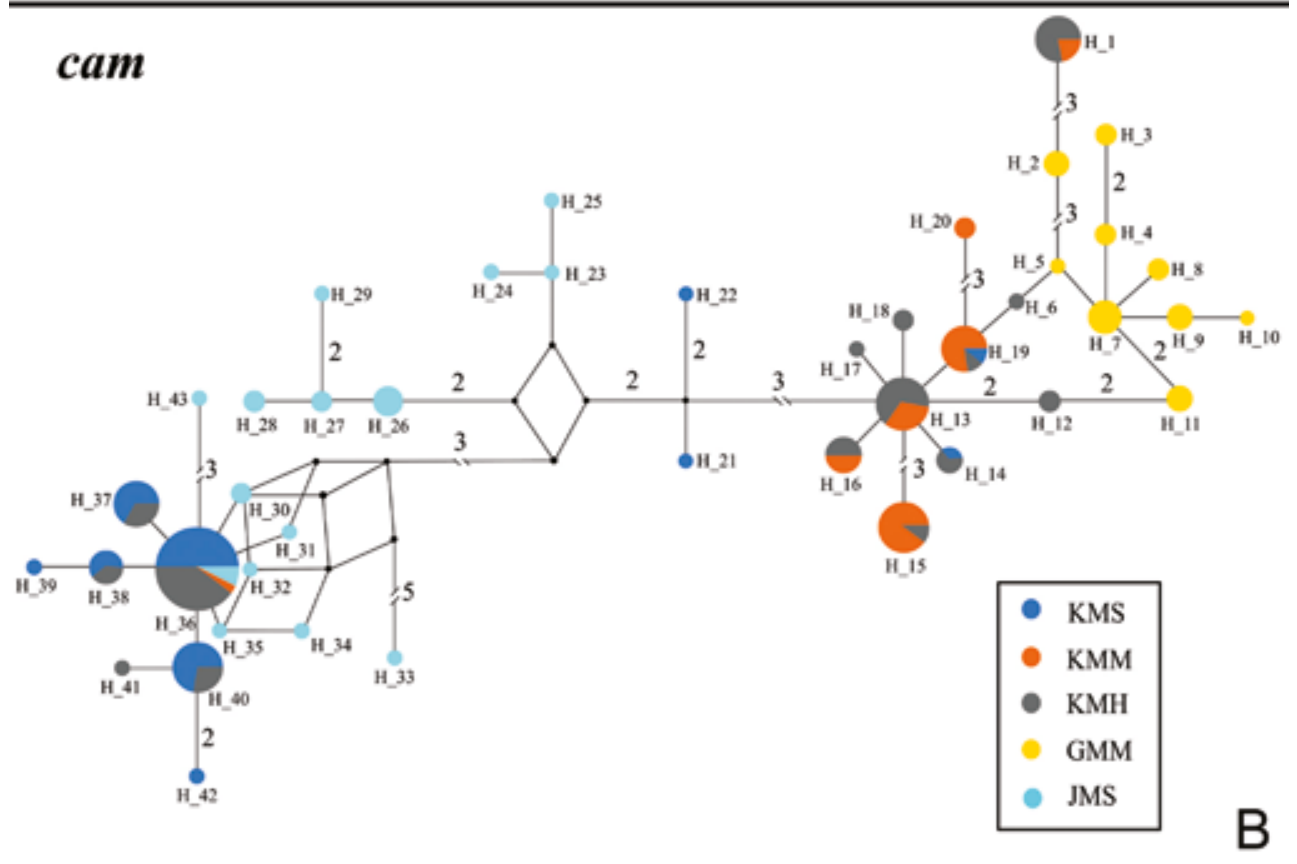

Fig. 3. Haplotype networks of the partial nuclear genes. A. tpi. B. cam. C. pcrf19 (continued on next page). Haplotypes of each population/taxon are shown in different colours. The sizes of the circles correspond to frequencies of the haplotypes. Small black circles represent hypothetical haplotypes. Mutational steps are shown by the number near the connecting lines, with the number omitted for those with only one mutation step. 


\section{pcrf1}

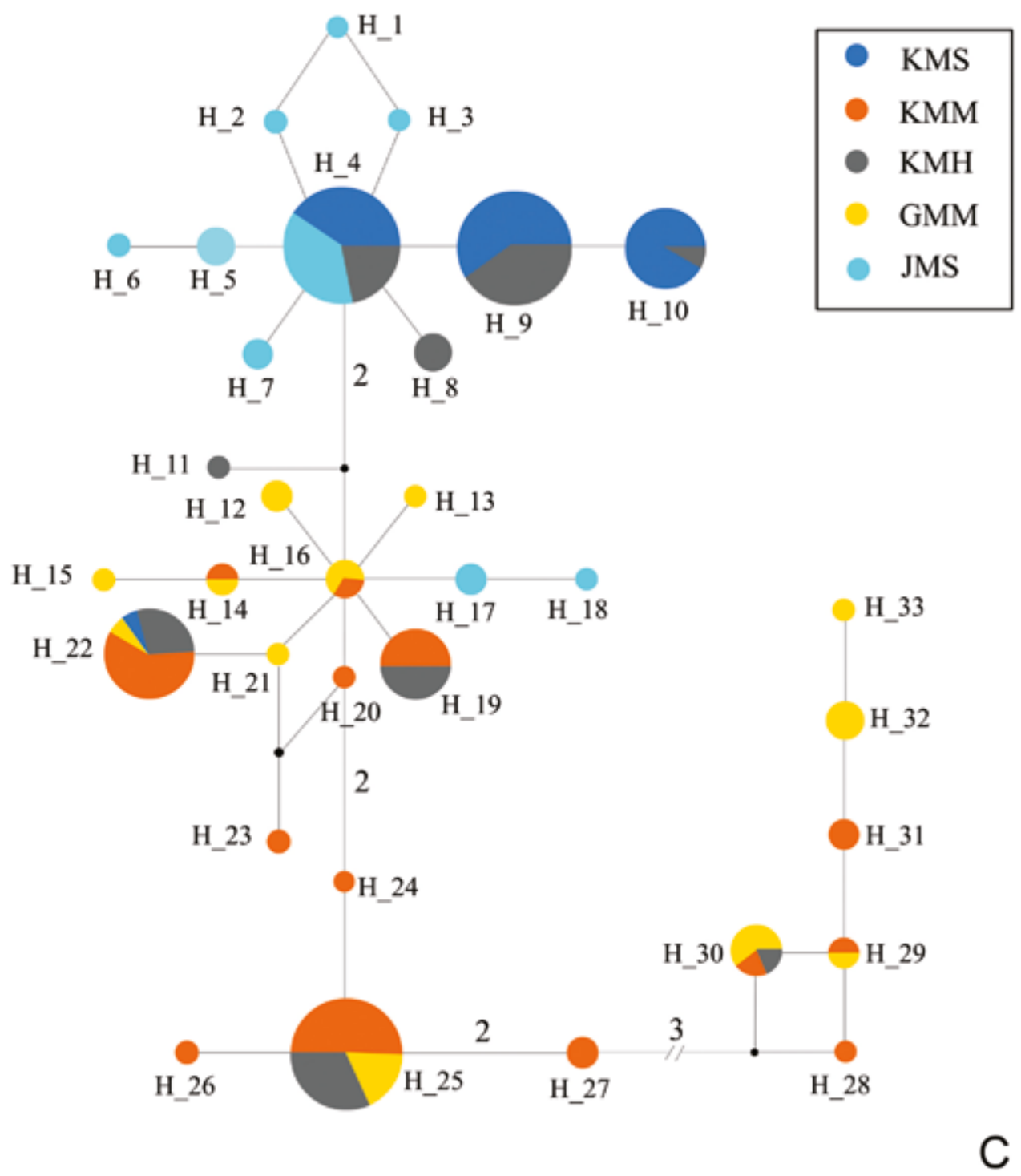

Fig. 3. (Continuation). Haplotype networks of the partial nuclear genes.

and M. malabathricum at one or more loci, supporting the hypothesis that they are hybrids between the two species. Moreover, most of the haplotypes (i.e. tpi: $95 \%$, cam: $82.5 \%$, pcrfl: $90 \%$, others were unique) harboured by these hybrids were shared with the parental individuals from the Kuala Selangor population, signifying that they are products of localised hybridisation. Such pattern of natural hybridisation is expected in Melastoma, as the member species are known to share pollinators, mainly small insects (Gross, 1993; Luo et al., 2008) that have limited pollination distances. 


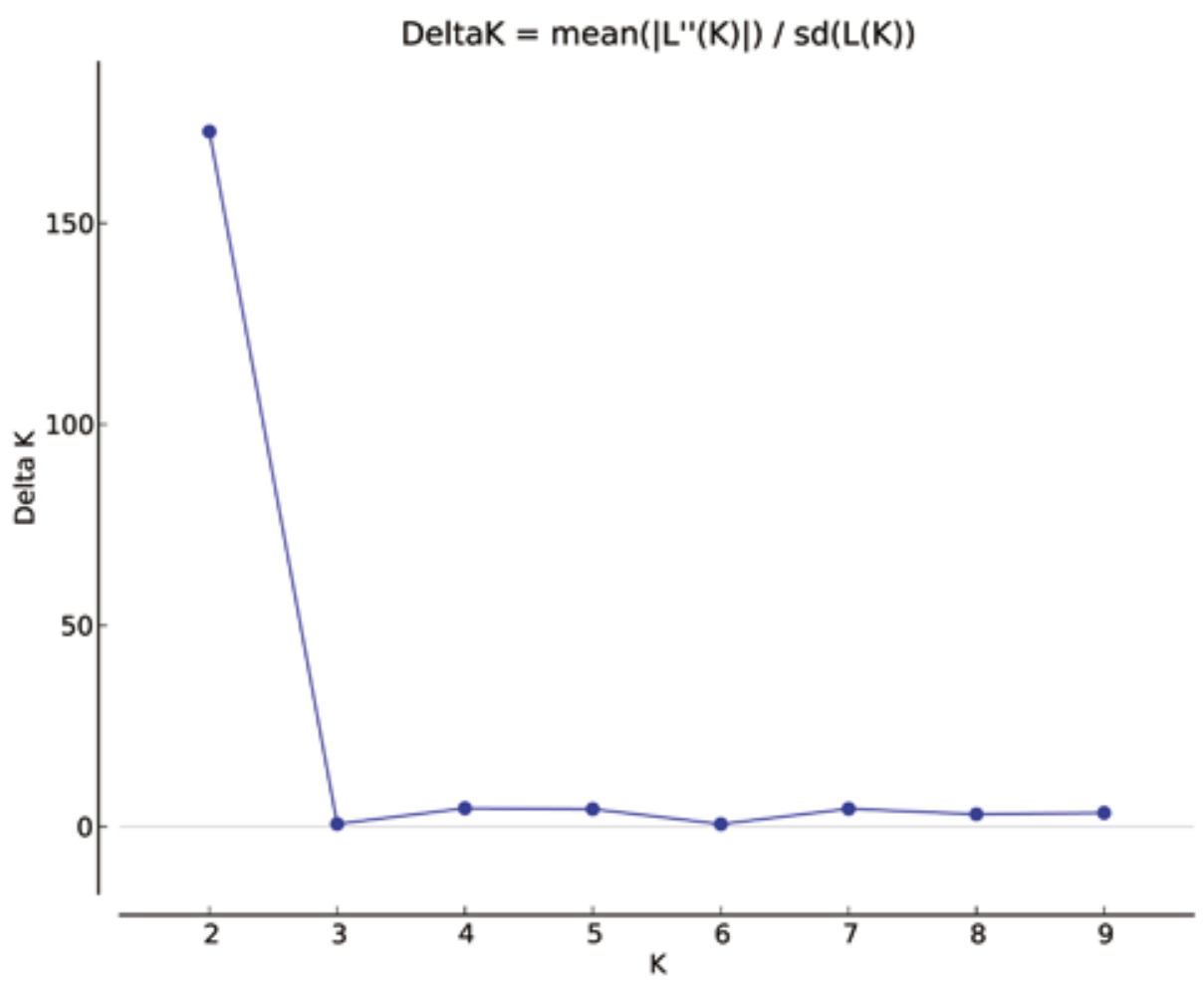

A

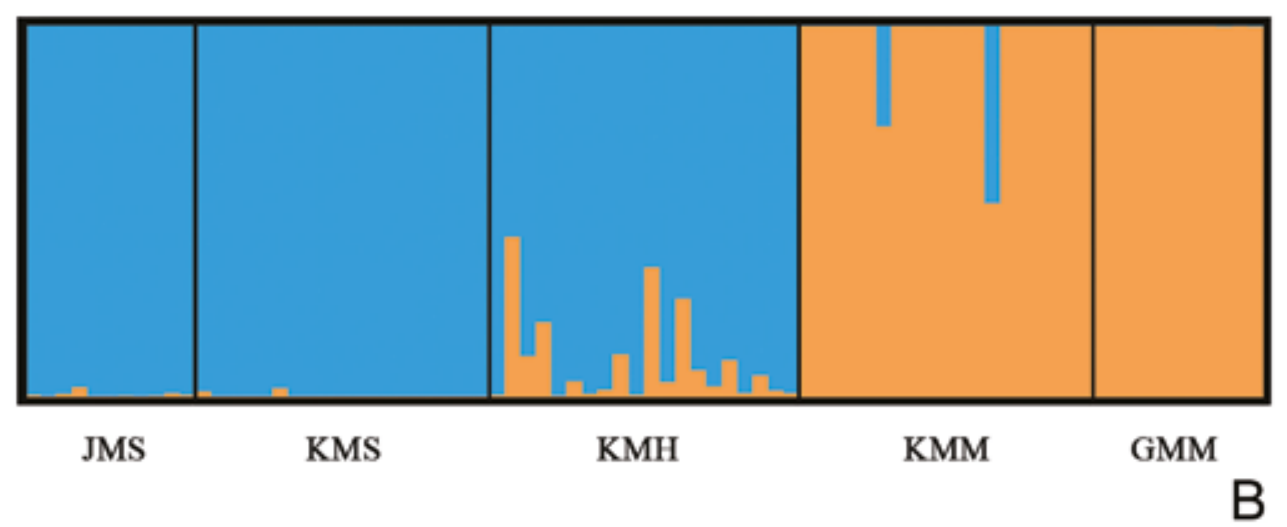

Fig. 4. Output of the Bayesian STRUCTURE analysis, showing: A. The highest $\Delta K$ value at $K$ $=2$. B. The bar plot of $K=2$.

Due to the fact that only one (out of 20) putative hybrid individual harboured haplotypes that fall under both clusters corresponding to the two parental species across all nuclear DNA loci (i.e. the signature of an F1 hybrid), we believe that hybridisation has been going on for more than one generation. This can be clearly observed from the results of the STRUCTURE analysis. The hybrid individuals also seem to mostly 
harbour a larger proportion of Melastoma sanguineum genetic component, although the reason for this bias is not immediately clear. Surprisingly, two individuals that were morphologically identified as Melastoma malabathricum (containing chloroplast haplotypes H_1 and H_2, respectively) turned out to contain significant proportions of $M$. sanguineum genetic component. Such an observation could be caused by introgression due to backcrossing or incomplete lineage sorting, although we believe introgression to be the main cause as it was only observed in the sympatric population. Finally, while the sympatric populations of Melastoma sanguineum and M. malabathricum each had its own specific chloroplast DNA haplotype (except for one individual identified as Melastoma sanguineum, which is also one of the suspected introgressants), the hybrid samples harboured both haplotypes. Thus, both species likely acted as the pollen donor in the hybridisation process.

To our knowledge, this study presents the first account of molecular evidence for natural hybridisation between Melastoma sanguineum and M. malabathricum. The fact that Southeast Asia harbours most of the Melastoma species diversity and that this phenomenon between two of the most widely distributed member species has been undocumented until now, goes to show that more effort is needed to study this unique group of plants.

ACKNOWLEDGEMENTS. This study was supported by grants from the National Natural Science Foundation of China (31670210 and 31811530297), Guangdong Natural Science Foundation (2015A030302011), Science and Technology Program of Guangzhou (201707010090), and Chang Hungta Science Foundation of Sun Yat-sen University.

\section{References}

Bandelt, H.J., Forster, P. \& Rohl, A. (1999). Median-joining networks for inferring intraspecific phylogenies. Molec. Biol. Evol. 16: 37-48.

Cai, Y., Wang F., Tan, G., Hu, Z., Wang, Y., Ng, W.L., Wu, W., Liu, Y. \& Zhou, R. (2019). Hybridization of Bornean Melastoma: implications for conservation of endemic plants in Southeast Asia. Bot. Lett. 166: 117-124.

Chao, L.Y., Chen, S., Wang, T., Liu, T., Wu, W., Dai, S., Wang, F., Fan, Q., Shi, S. \& Zhou, R. (2014). One species or two? Multilocus analysis of nucleotide variation of Melastoma penicillatum and Melastoma sanguineum (Melastomataceae) in Hainan, China. Biochem. Syst. Ecol. 55: 275-282.

Chen, J. (1983). On the genus Melastoma L. of Melastomataceae from China. J. South China Agric. Coll. 4: 31-36.

Chen, J. (1984). Melastomataceae. Flora Reipublicae Popularis Sinicae, vol. 53, pt. 1, pp. 152-161. Beijing: Science Press.

Dai, S., Wu, W., Zhang, R., Liu, T., Chen, Y., Shi, S. \& Zhou, R. (2012). Molecular evidence for hybrid origin of Melastoma intermedium. Biochem. Syst. Ecol. 41: 136-141.

Earl, D.A. \& vonHoldt, B.M. (2012). STRUCTURE HARVESTER: a website and program for visualizing STRUCTURE output and implementing the Evanno method. Conservation Genet. Resources 4: 359-361. 
Evanno, G., Regnaut, S. \& Goudet, J. (2005). Detecting the number of clusters of individuals using the software STRUCTURE: a simulation study. Molec. Ecol. 14: 2611-2620.

Gross, C.L. (1993). The breeding system and pollinators of Melastoma affine (Melastomataceae); a pioneer shrub in tropical Australia. Biotropica 25: 468-474.

Jakobsson, M. \& Rosenberg, N.A. (2007). CLUMPP: a cluster matching and permutation program for dealing with label switching and multimodality in analysis of population structure. Bioinformatics 23: 1801-1806.

Librado, P. \& Rozas, J. (2009). DnaSP v5: a software for comprehensive analysis of DNA polymorphism data. Bioinformatics 25: 1451-1452.

Liu, T., Chen, Y., Chao, L. Wang, S., Wu, W., Dai, S., Wang, F., Fan, Q. \& Zhou, R. (2014). Extensive hybridization and introgression between Melastoma candidum and $M$. sanguineum. PLoS ONE 9: e96690.

Luo, Z., Zhang, D. \& Renner, S.S. (2008). Why two kinds of stamens in buzz-pollinated flowers? Experimental support for Darwin's division-of-labour hypothesis. Funct. Ecol. 22: 794-800.

Meng, B.Y., Wakasugi, T. \& Sugiura, M. (1991). Two promoters within the psbK-psbI-trnG gene cluster in tobacco chloroplast DNA. Curr. Genet. 20: 259-264.

Meyer, K. (2001). Revision of Southeast Asian genus Melastoma (Melastomataceae). Blumea 46: 351-398.

Mogensen, H.L. (1996). The hows and whys of cytoplasmic inheritance in seed plants. Amer. J. Bot. 83: 383-404.

Pritchard, J.K., Stephens, M. \& Donnelly, P. (2000). Inference of population structure using multilocus genotype data. Genetics 155: 945-959.

Reginato, M. \& Michelangeli, F.A. (2016). Primers for low-copy nuclear genes in the Melastomataceae. Appl. Pl. Sci. 4: 1500092.

Renner, S.S. \& Meyer, K. (2001). Melastomeae come full circle: biogeographic reconstruction and molecular clock dating. Evolution 55: 1315-1324.

Rosenberg, N.A. (2004). DISTRUCT: a program for the graphical display of population structure. Molecular Ecology Notes 4: 137-138.

Wong, K.M. (2015). Studies in Southeast Asian Melastoma (Melastomataceae), 1. Morphological variation in Melastoma malabathricum and notes on rheophytic taxa and interspecific hybridisation in the genus. Gard. Bull. Singapore 67: 387-401.

Wong, K.M. (2016). The Genus Melastoma in Borneo including 31 new species. Kota Kinabalu: Natural History Publications, in association with National Parks Board, Singapore.

Wu, R., Zou, P., Tan, G., Hu, Z., Wang, Y., Ning, Z., Wu, W., Liu, Y., He, S. \& Zhou, R. (2019). Molecular identification of natural hybridization between Melastoma malabathricum and Melastoma beccarianum in Sarawak, Malaysia. Ecol. Evol. 9: 5766-5776.

Zhang, X., Dai, S., Jiang, J. \& Ma, G. (2010). The chromosome number and karyotypes of six species of Melastomataceae. J. Trop. Subtrop. Bot. 18: 386-390.

Zhou, Q., Cai, Y., Ng, W.L., Wu, W., Dai, S., Wang, F. \& Zhou, R. (2017). Molecular evidence for natural hybridization between two Melastoma species endemic to Hainan and their widespread congeners. Biodivers. Sci. 25: 638-646.

Zou, P., Ng, W.L., Wu, W., Dai, S., Ning, Z., Wang, S., Liu, Y., Fan, Q. \& Zhou, R. (2017). Similar morphologies but different origins: hybrid status of two more semi-creeping taxa of Melastoma. Front. Plant Sci. 8: 673. 
